\title{
Ações de vigilância à saúde integradas à Atenção Primária à Saúde diante da pandemia da COVID-19: contribuições para o debate
}

\author{
Primary care-based health surveillance actions in response \\ to the COVID-19 pandemic: contributions to the debate
}

Nilia Maria de Brito Lima Prado (https://orcid.org/0000-0001-8243-5662) ${ }^{1}$

Daniela Gomes dos Santos Biscarde (https://orcid.org/0000-0001-8758-2645) ${ }^{2}$

Elzo Pereira Pinto Junior (https://orcid.org/0000-0002-6977-2071) ${ }^{3}$

Hebert Luan Pereira Campos dos Santos (https://orcid.org/ 0000-0003-2722-7945) ${ }^{4}$

Sara Emanuela de Carvalho Mota (https://orcid.org/0000-0001-9502-5646) ${ }^{5}$

Erica Lima Costa de Menezes (https://orcid.org/0000-0002-3966-6382) ${ }^{6}$

Josilene Silva Oliveira (https://orcid.org/0000-0002-7857-7665) ${ }^{1}$

Adriano Maia dos Santos (https://orcid.org/0000-0001-9718-1562) ${ }^{1}$

${ }^{1}$ Instituto Multidisciplinar em Saúde, Universidade Federal da Bahia. Campus Anísio Teixeira. Rua Hormindo Barros 58 Quadra 17 Lote 58. 45029094 Vitória da Conquista. BA Brasil.

nilia.prado@ufba.br

${ }^{2}$ Escola de Enfermagem, Universidade Federal da Bahia. Salvador BA Brasil. ${ }^{3}$ Centro de Integração de Dados e Conhecimentos para a Saúde, Instituto Gonçalo Moniz, Fundação Oswaldo Cruz. Parque Tecnológico da Bahia. Salvador BA Brasil. ${ }^{4}$ Instituto Multidisciplinar em Saúde, Universidade Federal da Bahia. Vitória da Conquista BA Brasil.

${ }^{5}$ Superintendência Estadual, Ministério da Saúde na Bahia. Salvador BA Brasil. ${ }^{6}$ Núcleo Técnico Científico de Telessaúde, Diretoria de Atenção Básica, Secretaria de Saúde do Estado da Bahia. Salvador BA Brasil.
Abstract We conducted an integrated literature review aimed at reflecting on the challenges related to primary care-based health surveillance actions in response to the COVID-19 pandemic in selected countries. The study included countries with different PHC models that adopted surveillance as an approach to control the transmission of COVID-19. We performed a search in October 2020 for relevant literature and norms and guidelines related to the organization of primary health care (PHC) in response to the pandemic on official government websites and the databases Web of Science and Science Direct. The integrated health surveillance actions demonstrated that efforts were more focused on risks, with some countries adopting innovative and effective measures to respond to COVID-19, considering emerging needs within PHC. However, in addition to ethical controversies and operational difficulties, access to technology was a challenge in actions developed by some countries due to social inequalities.

Key words Coronavirus infection, Public health surveillance, Primary health care, Health policy
Resumo Trata-se de uma revisão de sintese integrativa com objetivo de refletir sobre os desafios atinentes às ações de vigilância em saúde no enfrentamento da COVID-19, no âmbito da Atenção Primária à Saúde (APS), em sistemas de saúde de países selecionados. Foram incluídos, no estudo, países com modelos de APS distintos, mas que adotaram a vigilância nos territórios como premissa para o controle da transmissão da COVID-19. Houve a revisão bibliográfica da literatura científica e a análise documental de normas e diretrizes relacionadas à organização da APS para enfrentamento da pandemia. A produção dos dados ocorreu no período entre abril e julho de 2020 e envolveu a busca de documentos sobre o enfrentamento da COVID-19, no que se refere à APS, nos sites oficiais governamentais de cada país e nas bases de dados científicas Web of Science e Science Direct. Ações integradas de vigilância em saúde demonstraram atuação mais direcionada sobre riscos, sendo possível respostas inovadoras e mais efetivas para enfrentamento da COVID-19, considerando necessidades emergentes no âmbito da APS. Contudo, experiências desenvolvidas por alguns países apresentaram controvérsias éticas e operacionais além dos desafios de acesso às tecnologias decorrente das desigualdades sociais.

Palavras-chave Infecção pelo coronavírus, Vigilância pública em saúde, Atenção Primária à Saúde, Política de saúde 


\section{Introdução}

Desde que a infecção causada pelo Coronavírus SARS-CoV-2 foi declarada pandemia, em 11 de março de 2020, pela Organização Mundial da Saúde (OMS), existem sérias preocupações sobre os efeitos deste vírus na saúde global, na sociedade e na economia, particularmente em populações vulneráveis e em países de baixa ou média renda com sistemas de saúde frágeis ${ }^{1,2}$.

Tal como aconteceu em surtos e pandemias anteriores, o controle da pandemia da COVID-19 depende da detecção oportuna dos casos e de seus contatos, seguida de medidas de isolamento e quarentena, com o objetivo de interromper a transmissão na comunidade e mitigar seus impactos na saúde humana ${ }^{3}$.

Globalmente, os países adotaram várias estratégias para enfrentar e controlar a pandemia da COVID-19, tais como testes na comunidade, rastreamento de contato, isolamento e outras medidas de saúde pública e sociais, cruciais para desacelerar a transmissão da doença e reduzir a mortalidade ${ }^{4}$. Neste contexto, uma vigilância em saúde robusta tem sido fundamental para controlar a disseminação e orientar a implementação contínua de medidas de prevenção.

A vigilância em saúde é apontada como uma função essencial em um sistema de saúde pública, para coleta, análise e interpretação sistemática contínua de dados aos tomadores de decisão, para planejamento e intervenção, em local adequado e tempo oportuno ${ }^{5}$. Outros autores referem como imprescindível para fornecer informações e orientar intervenções relacionadas ao diagnóstico e ao tratamento individual (enfoque clínico) ou direcionadas a grupos populacionais até a intervenção sobre os determinantes sociais em saúde (DSS) ${ }^{6,7}$. Esta função é mediada pela tradicional geração de dados da vigilância ou pela interpretação dos dados do sistema de vigilância, adaptados aos vários níveis do sistema de saúde, implementados para atender às necessidades e às dinâmicas cultural e populacional de cada país ${ }^{5}$.

Considerada a principal porta de entrada de uma pessoa no sistema de saúde, a Atenção Primária à Saúde (APS) é uma base crítica para a vigilância direta, com resposta oportuna e gestão de surtos. Com a expansão da pandemia e a incapacidade inicial de detectar e rastrear contatos para sua contenção, além de uma consequente flexibilização segura do isolamento social, a articulação entre vigilância em saúde pública e a APS constituiu-se em uma estratégia para garantir apoio técnico, operacional e logístico, bem como a provisão de recursos necessários para imple- mentar e desenvolver a base de um novo modus operandi, favorecendo maior participação social, otimização do uso dos equipamentos sociais e um processo eficaz de contenção da transmissão comunitária da COVID- $19^{8}$.

Uma variedade de modelos de trabalho utilizados em diversos surtos sanitários apoia a integração das funções de vigilância em saúde pública por médicos e equipes de saúde APS em zonas urbanas, rurais e remotas, com serviços coordenados e responsivos, em nível local e regional'. Dentre as estratégias, podem-se destacar os sistemas de agregação de dados, painéis de controle e a vigilância digital, entre outras. Estas fontes de dados vêm sendo cada vez mais integradas ao cenário de vigilância formal e têm um papel na vigilância da COVID-19 ${ }^{10,11}$. Tais iniciativas de monitoramento articulam-se a um conjunto de ações destinadas a controlar determinantes, riscos e danos à saúde de populações que vivem em determinados territórios, garantindo a integralidade da atenção, o que inclui tanto a abordagem individual como coletiva dos problemas de saúde ${ }^{12-14}$.

Nesse sentido, este artigo busca identificar e refletir sobre as experiências e limitações atinentes às distintas ações de vigilância em saúde a partir da APS, direcionadas ao enfrentamento da COVID-19, em sistemas de saúde de países selecionados.

\section{Aspectos metodológicos}

Trata-se de uma revisão de síntese integrativa, que abrange compilação de experiências relacionadas à vigilância em saúde para enfrentamento da COVID-19 no âmbito da APS, em alguns países atingidos pela pandemia.

Diante dos inúmeros desafios para a realização de estudos comparativos no campo das políticas de saúde, o risco de confusões semânticas, análises superficiais, descrições equivocadas, caricaturas, distorções retóricas e inferências injustificadas é frequente ${ }^{15}$. Ainda que constem apontamentos acerca de possíveis similaridades e divergências, optou-se pela síntese de ações derivadas de experiências de vigilância integrada internacionais no enfrentamento à COVID-19, sem uma perspectiva comparada e sem o intuito de replicá-las na realidade brasileira. Parte-se do pressuposto de que análises críticas e comparadas das respostas adotadas pelos diferentes sistemas de saúde requerem uma compreensão mais profunda dos contextos em que foram produzidas ${ }^{16}$.

Foram incluídas, no estudo, experiências desenvolvidas em países como África do Sul, Ar- 
gentina, Austrália, China, Coreia do Sul, Cuba, Estados Unidos, França, Itália, Índia Singapura e Reino Unido. Tratam-se de países com modelos de APS distintos, mas que adotaram, como premissa para o controle da transmissão da COVID-19, a vigilância ativa - intensiva e extensiva - nos territórios, direcionada para pessoas assintomáticas e sintomáticas, com apoio de profissionais de APS e da comunidade, e fluxo de encaminhamento coordenado aos demais serviços de urgência ou hospitalares. Excetuam-se os Estados Unidos, cujo modelo de atenção à saúde e de vigilância em saúde pública é fragmentado e distinto em cada estado, pois a forma de organização expressa respeito à autonomia de cada ente federado e seus condados. No entanto, isto resulta em baixa capacidade normativa e de coordenação da administração federal do país (Quadro 1).

A integração da APS com os serviços dos demais níveis de complexidade é uma característica essencial da APS abrangente, concepção aqui adotada. Além de sua integração no sistema de saúde, a articulação intersetorial é outro aspecto crucial para alcançar uma APS resolutiva.

A produção dos dados ocorreu entre abril e julho de 2020 e envolveu a busca de produções científicas sobre o enfrentamento da COVID-19, no escopo da APS, nas bases de dados científicas Web of Science e Science Direct, por meio da combinação dos descritores Health Primary Care AND Public Health Surveillance AND Coronavirus Infections. A coleta foi realizada nos meses de abril a outubro de 2020.

Os artigos em português, inglês e espanhol foram lidos no idioma original. Aqueles em chinês e francês foram traduzidos automaticamente para o inglês através da ferramenta Google Translate. Após as etapas de busca, seleção, leitura na íntegra e sistematização dos documentos, foram considerados os seguintes aspectos: autoria e ano de publicação, país de origem, título do documento e ações de vigilância em saúde adotadas (Quadro 2).

\section{Ações de vigilância em saúde pública}

As implicações decorrentes de emergências sanitárias têm grandes resultados para as populações dos locais e para os sistemas de vigilância dos serviços de saúde. A adoção de múltiplos mecanismos de vigilância pode garantir ampla cobertura, pois cada caso perdido pode levar a cadeias de transmissão que podem ser difíceis de serem contidos posteriormente.

No caso da pandemia em curso, os países variam amplamente em termos de sua capacidade de prevenir, detectar e responder a surtos, e em relação aos limites e às possibilidades entre a regulação exercida no nível central do sistema e o grau de autonomia e responsabilidade local. De maneira geral, as ações abrangem a vigilância em saúde pública, ativa e passiva, com algumas variações, ou um misto dos dois tipos, a depender dos arranjos tecnológicos possíveis na organização das práticas ${ }^{16,17}$.

Entre as abordagens de vigilância, a ativa abarca o monitoramento regular para buscar informações sobre as condições de saúde da população e fatores de risco comportamentais. É realizada pelos profissionais de saúde, com ou sem a participação comunitária, ou, ainda, com o apoio de Tecnologias de Informação e Cuidado em Saúde (TICS) e/ou intervenção mediada por canais de comunicação ${ }^{18}$. Já a vigilância passiva constitui um sistema pelo qual uma jurisdição de saúde recebe relatórios enviados por hospitais, clínicas, unidades de saúde pública ou outras fontes e os examina, bem como a história, rumores e outros dados sobre eventos de saúde, por meio de estratégias baseadas em unidades de saúde sentinelas, métodos agregados ou vigilância digital, mediante rastreamento de contatos ${ }^{19,20}$ e monitoramento passivo de dados de mídias sociais, para medir a atividade da doença (Figura 1).

$\mathrm{Na}$ China, o epicentro inicial da pandemia, foi utilizada a vigilância ativa, com atuação ativa dos médicos de APS, por meio de um trabalho de educação em saúde, com o apoio de Comitês Populares Locais para mobilizar moradores e voluntários, e monitorar os casos confirmados (monitoramento diário da situação de saúde e apoio psicológico para indivíduos sob quarentena $)^{21}$.Também foi utilizada vigilância passiva, com apoio de vigilância digital, para disponibilizar prescrições online, enviar mensagens instantâneas para transmissões comunitárias e painéis eletrônicos, a fim de disseminar informações e manter contato com centros especializados ambulatoriais ${ }^{22}$.

As redes lideradas pelos médicos de APS contribuíram significativamente para a coleta de dados e investigações epidemiológicas, através de comitês para triagem de saúde, rastreamento de contato e investigação de fontes de infecções, assim como do uso de interfaces mediante aplicativos de redes sociais para gerar uma combinação de dados agregados de saúde e uma classificação de risco de infecção pessoal ${ }^{23-25}$. Além disso, as ações incluíram a vigilância entre as pessoas que estavam em contato com a vida selvagem e expostas a fatores de risco comportamentais (alguns hábitos alimentares, como comer morcegos e outros $)^{26}$. 
Quadro 1. Características gerais dos sistemas de saúde e modalidade de organização da APS nos países selecionados.

\begin{tabular}{|c|c|c|c|c|}
\hline País & $\begin{array}{c}\text { Modalidade do } \\
\text { Sistema de Saúde }\end{array}$ & $\begin{array}{c}\text { Modalidade de Atenção } \\
\text { na APS }\end{array}$ & $\begin{array}{c}\text { Modalidade de } \\
\text { organização das } \\
\text { equipes na APS }\end{array}$ & $\begin{array}{l}\text { Resposta do primeiro nível } \\
\text { de atenção frente à epidemia } \\
\text { COVID-19 }\end{array}$ \\
\hline $\begin{array}{l}\text { África do } \\
\text { Sul }\end{array}$ & $\begin{array}{l}\text { Cobertura mista, } \\
\text { público e privado }\end{array}$ & $\begin{array}{l}\text { Sistema baseado em centros } \\
\text { comunitários de saúde, } \\
\text { com equipes compostas } \\
\text { por um profissional da } \\
\text { enfermagem e quatro a seis } \\
\text { agentes comunitários de } \\
\text { saúde. O médico de família } \\
\text { é multifacetado, sendo } \\
\text { essencial na porta de entrada } \\
\text { dos serviços de saúde bem } \\
\text { como na administração de } \\
\text { qualidade. Desenvolvimento } \\
\text { contínuo dos profissionais, } \\
\text { aplicação de diretrizes, } \\
\text { tratamento e protocolo das } \\
\text { condições de saúde }\end{array}$ & $\begin{array}{l}\text { A atuação das equipes } \\
\text { está centrada na } \\
\text { realização de visitas } \\
\text { domiciliares e busca } \\
\text { ativa de pacientes, com } \\
\text { abordagem generalista } \\
\text { e ênfase na atenção } \\
\text { às doenças mais } \\
\text { prevalentes. Reconhece } \\
\text { a importância } \\
\text { da participação } \\
\text { comunitária, que tem } \\
\text { direito de planejar } \\
\text { e implementar seus } \\
\text { próprios programas e } \\
\text { serviços de saúde }\end{array}$ & $\begin{array}{l}\text { Disponibilização de } 28 \\
\text { mil profissionais de saúde } \\
\text { comunitários para realizar } \\
\text { busca ativa de casa em casa nas } \\
\text { comunidades com maior risco de } \\
\text { contaminação e a realização das } \\
\text { visitas domiciliares }\end{array}$ \\
\hline Argentina & $\begin{array}{l}\text { Sistema de } \\
\text { saúde misto, } \\
\text { em três grandes } \\
\text { subsistemas: } 1 . \\
\text { Público, universal } \\
\text { e gratuito; } 2 . \\
\text { Seguridade Social, } \\
\text { com investimentos } \\
\text { públicos, dos } \\
\text { trabalhadores; } \\
\text { 3. Sistema } \\
\text { privado de saúde } \\
\text { envolve serviços } \\
\text { corporativos e } \\
\text { medicina pré-paga }\end{array}$ & $\begin{array}{l}\text { Em geral, a Atenção } \\
\text { Primária à Saúde é prestada } \\
\text { por centros e postos de } \\
\text { saúde sob gestão municipal } \\
\text { e provincial sendo afirmada } \\
\text { como porta de entrada } \\
\text { preferencial e filtro para a } \\
\text { atenção especializada }\end{array}$ & $\begin{array}{l}\text { Modelo centrado nas } \\
\text { especialidades }\end{array}$ & $\begin{array}{l}\text { Implantação do serviço Tele- } \\
\text { Covid com cobertura exclusiva } \\
\text { do sistema público para que } \\
\text { possam fazer consultas de saúde } \\
\text { com especialistas de suas casas } \\
\text { por meio de videochamadas. } \\
\text { Uso da ferramenta Tele-Covid } \\
\text { para rastrear casos suspeitos } \\
\text { ou confirmados de COVID-19, } \\
\text { a fim de impedir a circulação } \\
\text { de pessoas e para compartilhar } \\
\text { conhecimentos, experiências } \\
\text { de atendimento e coordenar } \\
\text { ações entre o sistema de APS, } \\
\text { emergência e o hospital, } \\
\text { laboratórios. } \\
\text { Uso de dispositivo de teste } \\
\text { estratégico para coronavírus no } \\
\text { campo da Argentina (DETeCTAr) }\end{array}$ \\
\hline Austrália & $\begin{array}{l}\text { Cobertura mista, } \\
\text { público (Medicare- } \\
\text { universal) e privada }\end{array}$ & $\begin{array}{l}\text { A APS é a porta de } \\
\text { entrada, fornecida em } \\
\text { domicilio ou em ambientes } \\
\text { comunitários, em } \\
\text { práticas gerais, privadas, } \\
\text { saúde da comunidade, } \\
\text { governo local e serviços } \\
\text { não governamentais. } \\
\text { Os Medicare Locals e } \\
\text { Local Hospital Networks } \\
\text { trabalham como } \\
\text { consumidores e provedores } \\
\text { locais para desenvolver } \\
\text { planos e serviços } \\
\text { integrados, coordenados e } \\
\text { responsivos em nível local }\end{array}$ & $\begin{array}{l}\text { Equipe } \\
\text { multiprofissional }\end{array}$ & $\begin{array}{l}\text { As pessoas mais vulneráveis } \\
\text { recebem seus cuidados médicos } \\
\text { e conselhos da atenção primária, } \\
\text { geralmente através da prática } \\
\text { geral das equipes do Medicare. } \\
\text { Treinamento online para os } \\
\text { profissionais de saúde. Prestação } \\
\text { de aconselhamento e apoio ao } \\
\text { público sobre distanciamento } \\
\text { social; investimento substancial } \\
\text { em apoio à saúde mental para } \\
\text { todos os australianos; assistência } \\
\text { a idosos, serviços para deficientes } \\
\text { e hospitais; Teleconsulta, } \\
\text { triagem, prescrição eletrônica, } \\
\text { telemonitoramento }\end{array}$ \\
\hline
\end{tabular}


Quadro 1. Características gerais dos sistemas de saúde e modalidade de organização da APS nos países selecionados.

\begin{tabular}{|c|c|c|c|c|}
\hline País & $\begin{array}{l}\text { Modalidade do } \\
\text { Sistema de Saúde }\end{array}$ & $\begin{array}{c}\text { Modalidade de Atenção } \\
\text { na APS }\end{array}$ & $\begin{array}{l}\text { Modalidade de } \\
\text { organização das } \\
\text { equipes na APS }\end{array}$ & $\begin{array}{c}\text { Resposta do primeiro nível } \\
\text { de atenção frente à epidemia } \\
\text { COVID-19 }\end{array}$ \\
\hline China & $\begin{array}{l}\text { Cobertura mista, } \\
\text { público e privado }\end{array}$ & $\begin{array}{l}\text { Todo cidadão tem direito a } \\
\text { receber serviços básicos de } \\
\text { saúde, pelos governos locais }\end{array}$ & $\begin{array}{l}\text { Principalmente } \\
\text { médicos da aldeia e } \\
\text { profissionais de saúde } \\
\text { em clínicas rurais, } \\
\text { clínicos gerais (GPs) } \\
\text { em municípios rurais e } \\
\text { hospitais comunitários } \\
\text { urbanos e profissionais } \\
\text { médicos em hospitais } \\
\text { secundários e terciários }\end{array}$ & $\begin{array}{l}\text { Ampla atuação dos GP } \\
\text { envolvidos no diagnóstico e } \\
\text { tratamento e educação em } \\
\text { saúde, vigilância ativa e passiva, } \\
\text { monitoramento e orientações/ } \\
\text { atendimentos. GP atuam em } \\
\text { conjunto com o bairro local, } \\
\text { comitês e polícia comunitária } \\
\text { em uma defesa conjunta, para } \\
\text { aconselhamento, triagem e } \\
\text { monitoramento. WeChat - } \\
\text { Redes de triagem de saúde que } \\
\text { podem fornecer rastreamento de } \\
\text { contatos e investigação das fontes } \\
\text { de infecções }\end{array}$ \\
\hline $\begin{array}{l}\text { Coreia do } \\
\text { Sul }\end{array}$ & $\begin{array}{l}\text { Cobertura mista, } \\
\text { público e privado }\end{array}$ & $\begin{array}{l}\text { Os médicos atendem em } \\
\text { consultórios privados }\end{array}$ & $\begin{array}{l}\text { Combinação de tarefas } \\
\text { profissional na equipe } \\
\text { entre as categorias } \\
\text { de enfermagem e } \\
\text { assistentes sociais }\end{array}$ & $\begin{array}{l}\text { Diagnósticos massivos para a } \\
\text { doença, obtidos por meio de } \\
\text { uma política de testar o maior } \\
\text { número possível de pessoas e uso } \\
\text { de tecnologia para rastreamento } \\
\text { de casos }\end{array}$ \\
\hline EUA & $\begin{array}{l}\text { Não possui } \\
\text { sistema universal. } \\
\text { Assistência por } \\
\text { seguro de saúde } \\
\text { privado e cobertura } \\
\text { de saúde pública }\end{array}$ & $\begin{array}{l}\text { Os cuidados médicos } \\
\text { primários nos EUA } \\
\text { são prestados por três } \\
\text { especialidades: medicina de } \\
\text { família, medicina interna } \\
\text { geral e pediatria geral }\end{array}$ & $\begin{array}{l}\text { Os médicos, cuja } \\
\text { função principal } \\
\text { é direcionar o } \\
\text { atendimento ao } \\
\text { paciente, além de } \\
\text { enfermeiros e médicos } \\
\text { assistentes }\end{array}$ & $\begin{array}{l}\text { Baixa organização dos serviços } \\
\text { de APS; tentativa de organização } \\
\text { de Medicare e Medicaid; } \\
\text { Isolamento de contatos e casos } \\
\text { confirmados com confirmação } \\
\text { de testes laboratoriais; Serviços } \\
\text { de urgência e emergência } \\
\text { pagos pelos usuários mediante } \\
\text { necessidade de uso; Suporte } \\
\text { disponível de outras agências } \\
\text { governamentais estaduais / } \\
\text { locais e organizações parceiras; } \\
\text { Dificuldade para implantar a } \\
\text { telemedicina; seguros privados } \\
\text { instituíram apenas para idosos } \\
\text { segurados }\end{array}$ \\
\hline França & Universal & $\begin{array}{l}\text { Os médicos de clínica geral } \\
\text { trabalham em consultório } \\
\text { particular ou em centros } \\
\text { de saúde }\end{array}$ & $\begin{array}{l}\text { A maioria dos cuidados } \\
\text { ambulatoriais prestados } \\
\text { pelos enfermeiros em } \\
\text { casa, especialmente } \\
\text { para pacientes idosos } \\
\text { e deficientes. Os } \\
\text { profissionais } \\
\text { paramédicos } \\
\text { incluem os dentistas, } \\
\text { farmacêuticos, } \\
\text { fisioterapeutas } \\
\text { e parteiras e } \\
\text { especialistas em } \\
\text { ambulatório, pediatras, } \\
\text { ginecologistas, } \\
\text { oftalmologistas e } \\
\text { psiquiatras }\end{array}$ & $\begin{array}{l}\text { Hierarquização funcional em } \\
\text { dois níveis das unidades de saúde; } \\
\text { incentivo com dobro do valor; } \\
\text { consultas aos GPs; ampliação } \\
\text { da telemedicina. Medicina } \\
\text { ambulatorial da cidade e outras } \\
\text { unidades de saúde continuem } \\
\text { funcionando normalmente }\end{array}$ \\
\hline
\end{tabular}


Quadro 1. Características gerais dos sistemas de saúde e modalidade de organização da APS nos países selecionados.

\begin{tabular}{|c|c|c|c|c|}
\hline País & $\begin{array}{l}\text { Modalidade do } \\
\text { Sistema de Saúde }\end{array}$ & $\begin{array}{c}\text { Modalidade de Atenção } \\
\text { na APS }\end{array}$ & $\begin{array}{c}\text { Modalidade de } \\
\text { organização das } \\
\text { equipes na APS }\end{array}$ & $\begin{array}{l}\text { Resposta do primeiro nível } \\
\text { de atenção frente à epidemia } \\
\text { COVID-19 }\end{array}$ \\
\hline Itália & Universal & $\begin{array}{l}\text { O modelo GP e algumas } \\
\text { equipes interprofissionais } \\
\text { ambulatoriais }\end{array}$ & $\begin{array}{l}\text { Predomina o GP e } \\
\text { em algumas regiões, } \\
\text { a colaboração } \\
\text { interprofissional } \\
\text { para melhorar a } \\
\text { acessibilidade, equidade } \\
\text { e continuidade do } \\
\text { cuidado }\end{array}$ & $\begin{array}{l}\text { Descentralização dos serviços de } \\
\text { saúde e assistência da população } \\
\text { potencialmente afetada; } \\
\text { Recrutamento de profissionais } \\
\text { de saúde para as regiões mais } \\
\text { atingidas (Lombardia). Parcerias } \\
\text { sólidas entre os setores público e } \\
\text { privado foram institucionalizadas. } \\
\text { Teleconsulta; prescrição eletrônica } \\
\text { interligada as farmácias }\end{array}$ \\
\hline Índia & $\begin{array}{l}\text { Misto, público } \\
\text { e privado. A } \\
\text { responsabilidade } \\
\text { dos estados, que } \\
\text { muitas vezes } \\
\text { são incapazes de } \\
\text { garantir cobertura } \\
\text { em regiões } \\
\text { vulneráveis ou } \\
\text { isoladas }\end{array}$ & $\begin{array}{l}\text { Os cuidados para os } \\
\text { pobres são prestados por } \\
\text { prestadores, comerciais } \\
\text { ou sem fins lucrativos, } \\
\text { muitas vezes sem licença, } \\
\text { em grande parte não } \\
\text { regulamentados }\end{array}$ & $\begin{array}{l}\text { As policlínicas se } \\
\text { consolidaram como } \\
\text { uma alternativa ao } \\
\text { sistema capitalista } \\
\text { de prestação de } \\
\text { serviços de saúde, } \\
\text { oferecendo tratamentos } \\
\text { de forma acessível para } \\
\text { a população e também } \\
\text { residências médicas } \\
\text { em clínica geral. } \\
\text { Cuidados ofertados } \\
\text { para as populações } \\
\text { desemparadas, com } \\
\text { especial atenção às } \\
\text { mulheres e crianças }\end{array}$ & $\begin{array}{l}\text { Programa de Vigilância Integrada } \\
\text { de Doenças por meio dos dados } \\
\text { de localização do telefone e } \\
\text { Bluetooth; atualizações contínuas } \\
\text { do Ministério da Saúde e } \\
\text { números de linhas telefônicas } \\
\text { de ajuda. Dificuldades para a } \\
\text { população adotar medidas de } \\
\text { higiene e distanciamento social }\end{array}$ \\
\hline $\begin{array}{l}\text { Reino } \\
\text { Unido }\end{array}$ & Universal & $\begin{array}{l}\text { GP são a porta de entrada } \\
\text { do sistema de saúde }\end{array}$ & $\begin{array}{l}\text { A equipe principal de } \\
\text { cuidados primários } \\
\text { inclui médicos } \\
\text { de clínica geral } \\
\text { (GPs), enfermeiros } \\
\text { profissionais s e } \\
\text { equipe gerencial / } \\
\text { administrativa, ligados } \\
\text { à clínica ou a uma } \\
\text { população definida } \\
\text { geograficamente }\end{array}$ & $\begin{array}{l}\text { Triagem de todos os pacientes } \\
\text { remotamente por uma equipe de } \\
\text { saúde, considerando os pacientes } \\
\text { suspeitos, os grupos de risco } \\
\text { e a ampla população; grupo } \\
\text { de comissionamento clínico } \\
\text { (CCG); instituiu um programa } \\
\text { de treinamento de emergência } \\
\text { em larga escala para recrutar } \\
\text { trabalhadores comunitários; } \\
\text { ampliação da teleconsulta e } \\
\text { telemonitoramento }\end{array}$ \\
\hline Singapura & $\begin{array}{l}\text { O sistema de } \\
\text { saúde do governo } \\
\text { baseia-se no quadro } \\
\text { " } 3 \mathrm{M} \text { ": o Medifund, } \\
\text { a Medisave, } \\
\text { Medishield }\end{array}$ & $\begin{array}{l}\text { Os cuidados primários são } \\
\text { a base do sistema de saúde, } \\
\text { prestados por policlínicas } \\
\text { do governo e clínicas } \\
\text { privadas de GP }\end{array}$ & $\begin{array}{l}\text { Os cuidados primários } \\
\text { são prestados por } \\
\text { meio de uma rede de } \\
\text { policlínicas ambulatoriais } \\
\text { e clínicas administradas } \\
\text { por clínicos gerais (GPs) } \\
\text { apoiados por Clínicas } \\
\text { de Medicina Familiar } \\
\text { (FMCs). E as equipes } \\
\text { dos Centros de Saúde } \\
\text { Comunitários (CHCs) } \\
\text { que compõem as Redes } \\
\text { de Atenção Básica (PCN) }\end{array}$ & $\begin{array}{l}\text { Exames e atendimento médico } \\
\text { gratuitos a todos os cidadãos } \\
\text { durante o surto; sistema de } \\
\text { rastreamento de contato rígido, } \\
\text { com isolamento mediado } \\
\text { por acusações criminais para } \\
\text { aqueles que violassem ordens; } \\
\text { distanciamento social e } \\
\text { pagamento de multas até US\$ } 7 \\
\text { mil; atendimento hospitalar nos } \\
\text { hospitais públicos organizados } \\
\text { e com recursos do Ministério da } \\
\text { Saúde. Monitoramento mediado } \\
\text { por tecnologia da informação }\end{array}$ \\
\hline
\end{tabular}




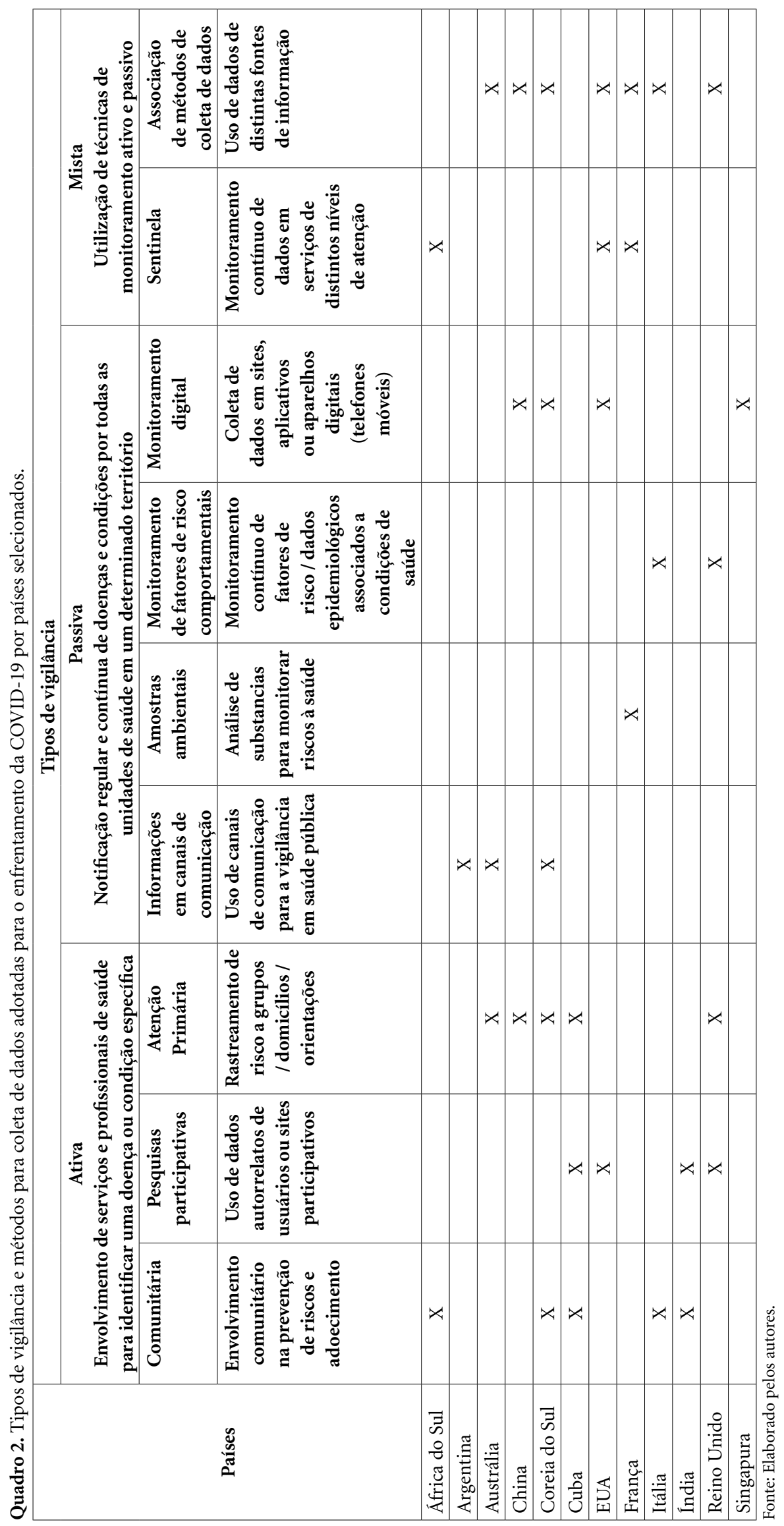




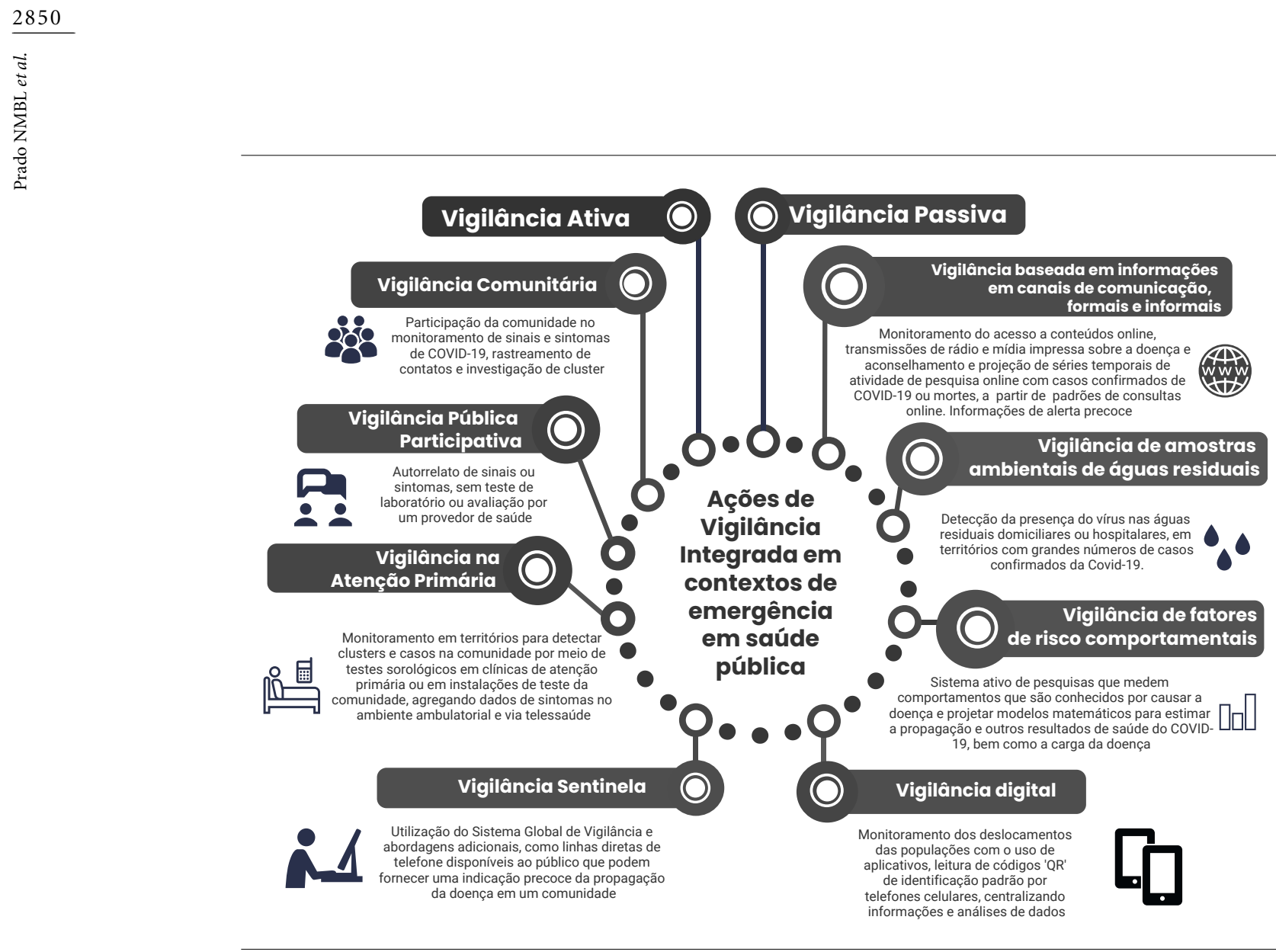

Figura 1. Ações de vigilância integrada em contextos de emergência em saúde pública.

Fonte: Elaborado pelos autores.

Singapura priorizou a vigilância passiva por meio de metodologias aprimoradas de rastreamento de contato adotadas e praticadas regularmente, além de um sistema de rastreamento que podia identificar, relatar a localização de pessoas em quarentena via global positioning system (GPS) e vincular essas informações aos resultados de seus testes sorológicos, permitindo a obtenção de um mapa da cadeia de transmissão e o compartilhamento de informações entre os serviços de epidemiologia das doenças infecciosas derivados de experiências anteriores com surtos respiratórios ${ }^{27}$.

Na Coréia do Sul, todos os serviços de APS deviam realizar triagem ativa por meio de busca ativa nos domicílios dos usuários, e passiva, por telerrastreio ${ }^{28}$. Os usuários com triagem positiva por análise clínico-epidemiológica eram encaminhados aos centros de saúde para consulta presencial, testagem e diagnóstico. Posteriormente, eram monitorados diariamente (casos confirmados, prováveis e suspeitos) por meio de uma ava- liação de risco individual, conduzida por médicos de APS, para determinar o nível e os parâmetros necessários de quarentena e isolamento. Os atendimentos na APS estavam integrados à vigilância em saúde e forneciam a situação epidemiológica local e nacional ${ }^{29}$.

Nos Estados Unidos, a vigilância em saúde foi desenvolvida pelos Centros de Controle e Prevenção de Doenças (CDC) e pelo Sistema de Doenças Infecciosas Emergentes Globais, do Departamento de Defesa (USDOD). Durante a pandemia, foram utilizados o ILINet, um sistema de vigilância para doenças semelhantes à Influenza, assim como as bases de dados ProMed, Epi-X e relatórios derivados do aplicativo COVID Near You. Contudo, embora informativos, estes sistemas podem apresentar viés de seleção, interpretação excessiva dos resultados devido à falta de integração com a vigilância oficial, dada a fragmentação do sistema de saúde e da APS, bem como a tomada de decisões distintas pelos gestores de cada Estado que compõem o país ${ }^{30}$. 
A Itália optou pela vigilância passiva, por meio de dados de vigilância integrada da $\mathrm{CO}$ VID-19 ${ }^{31}$ recolhidos pelo Instituto Superiore di Sanità (ISS), através de plataforma web exclusiva, questionários eletrônicos e infográficos diários, processados exclusivamente para fins de vigilância epidemiológica e microbiológica no contexto da epidemia de SARS-CoV-2. Os dados permitiram traçar o quadro epidemiológico nacional e dos sistemas regionais de saúde (órgãos e autoridades em todo o território nacional, tais como regiões, autarquias locais e regionais de saúde) $)^{31,32}$.

As equipes multiprofissionais de APS do Medicare Locals, na Austrália, priorizaram o rastreamento de contatos e grupos vulneráveis - especialmente, os idosos - e o monitoramento de usuários com sintomas leves de doenças respiratórias (vigilância sindrômica) nas comunidades ${ }^{33}$. Em nível nacional, o FluTracking, um sistema de vigilância interligado, permite uma abordagem coordenada e consistente para a vigilância da COVID-19, com dados derivados da Rede de Pesquisa de Práticas Sentinela (ASPREN) e do Victoria Sentinel Practice Influenza Network (VicSPIN), sistema de vigilância sentinela de médicos generalistas e de médicos da Commonwealth Clínicas Respiratórias $^{34}$. A vigilância incluiu a população aborígene e os residentes das ilhas do Estreito de Torres $^{35}$.

A África do Sul elegeu a vigilância ativa, baseada na comunidade, para detecção precoce, confirmação rápida, rastreamento de contato, montagem de centros de isolamento de pessoal e apoio ao cuidado, por meio de Vigilância e Resposta Integrada a Doenças (IDSR), que fornece estrutura para uma vigilância sindrômica, identificando o ponto de entrada para verificar, caracterizar e responder à transmissão da COVID-19 na comunidade ${ }^{36}$. Além disso, foram utilizados os sistemas da vigilância demográfica implantada pelo Africa Health Research Institute (AHRI) para realizar a vigilância comunitária da COVID-19 em ambiente rural. Esses sistemas de coleta de dados constituíram uma ferramenta vital para a triagem dos sintomas da COVID-19 em cada membro residente das famílias e na resposta da saúde pública à COVID-19 em áreas remotas ${ }^{37-38}$.

A Índia, com um bom suporte de telecomunicações, mas recursos escassos e um sistema de saúde não tão robusto, apresenta um sistema de vigilância participativa, denominado Aarogya Setu (que se traduz, do sânscrito, como "uma ponte de saúde"), para a prevenção e o controle da pandemia, com participação voluntária dos usuários ${ }^{39}$.Este sistema complementa o Programa de Vigilância Integrada de Doenças por meio dos dados de localização do telefone e Bluetooth, para avaliar a proximidade de uma pessoa infectada pelo SARS-CoV-2, comparando bancos de dados de casos confirmados de COVID-19 em laboratório para classificar o status de risco do indivíduo como baixo, moderado ou alto ${ }^{40}$.Os indivíduos eram informados sobre as medidas a serem tomadas com base na avaliação de risco e aconselhamento para teste, com detalhes de centros de teste disponíveis em suas áreas. Eles também contaram com um recurso de chatbot, atualizações contínuas do Ministério da Saúde e números de linhas telefônicas de ajuda, em cada estado do país ${ }^{40,41}$.

Na França, o uso da Rede Sentinela pelos médicos da APS foi apoiado com a implantação de aplicativos com linguagem Java instalados nos computadores das unidades de saúde, que permitem notificar os casos, implementar protocolos de vigilância e questionários, bem como instâncias de descrições de casos em regiões com baixo acesso à rede de internet, pois estão interligados a um servidor principal, nos centros de vigilância nacional ${ }^{42}$.

No Reino Unido, o National Health Service e a Public Health England têm um sistema de vigilância comunitária interligado ao sistema de vigilância sindrômica, com monitoramento por uma equipe de vigilância em tempo real (ReSST) do sistema de saúde, coordenando vários subsistemas nacionais e viabilizando as transmissões semanais de dados do Royal College of General Practitioners (RCGP), administrado pelo RCGP Research and Surveillance Center (RCGP RSC) ${ }^{11}$. O sistema de vigilância virtual usa estatísticas de frequência de consulta de pesquisa diária extraídas da API Google Health Trend, geradas por usuários, sobre os sintomas, bem como modelos para monitoramento da COVID-1943 baseados em algoritmos de pesquisa da internet previamente estabelecidos para Influenza (FluSurvey), mas atualmente incluídos nos relatórios semanais da Public Health England para a COVID-19 $9^{44,45}$. Isto permite a análise de dados de saúde anônimos, de várias fontes, e favorece a procura por tendências que indiquem níveis de doença acima do normal, divulgando boletins para manter os profissionais de saúde pública atualizados, sempre agregando dados de sintomas no ambiente ambulatorial ${ }^{44,45}$.

O Ministério da Saúde da Argentina, como esforço para combater e conter o vírus, implementou um plano de rastreamento de contatos chamado Detectar, em áreas definidas, nas quais é detectado ou estimado o aumento do número de casos. Lançado em maio, depois de um grande 
salto no número de casos na baixa classe socioeconômica e em bairros densamente povoados da grande Buenos Aires, conhecidos como vilas, o plano foi posteriormente estendido por todo o país ${ }^{46-48}$.

Em Cuba, a experiência anterior com epidemias (Dengue e Zika), abordada no contexto de um sistema universal de saúde, foi uma vantagem. Já existia uma rede nacional de diagnóstico e vigilância, apoiada pelos Centros Provinciais de Higiene, Epidemiologia e Microbiologia, uma rede nacional de laboratórios de diagnóstico em conformidade com a OMS e o laboratório nacional do Centro de Referência para Doenças Infecciosas, no Instituto de Medicina Tropical Pedro Kourí (IPK), de Havana ${ }^{49,50}$. O Ministério da Saúde cubano, antes do primeiro caso, capacitou todos os seus profissionais para o manejo e a vigilância comunitária ${ }^{49,50}$.

A equipe de APS operou sob protocolos para avaliações contínuas de saúde comunitária (saúde pública-epidemiologia), bem como para avaliações de saúde individual e familiar para detecção de sintomas respiratórios, por meio de atividades diárias ativas em todos os bairros cubanos, envolvendo rastreamento de casos suspeitos e confirmados, com a participação de aproximadamente 28 mil estudantes de medicina, residentes voluntários ${ }^{49,50}$. Utilizaram-se as visitas domiciliares para ampliar o monitoramento dos usuários com alto risco e os casos confirmados em isolamento domiciliar, mediante sucessivos exames físicos completos e uma avaliação abrangente, com ênfase nos grupos vulneráveis ${ }^{50,51}$.

Um tipo de vigilância ainda pouco utilizado, a detecção de SARS-CoV-2 em uma série de amostras longitudinais de águas residuais metropolitanas coletadas durante os estágios iniciais da epidemia foi utilizado em países como Espanha e França, conseguindo identificar RNA viral de águas residuais correlacionado ao aumento do número de casos declarados ${ }^{52}$. A análise rotineira de águas residuais representa uma estratégia sensível e econômica para a vigilância da COVID-19, favorecendo uma melhora significativa na preparação dos sistemas de saúde contra surtos virais novos ou recorrentes ${ }^{53}$.

\section{Desafios, limitações e considerações finais}

A vigilância pública em saúde constitui um mecanismo essencial para a compreensão da epidemiologia da doença e fornece uma base sólida para a implementação de medidas de controle.
A maioria dos países selecionados adotou diferentes abordagens prioritárias para a vigilância em saúde pública, abrangendo registros sistemáticos de condições de saúde prevalentes, obtidos por sistemas de dados administrativos, dados de estatísticas vitais e pesquisas anuais de saúde. Contudo, as abrangências das ações de vigilância da saúde pública podem apresentar fragilidades, mesmo em países com sistemas de saúde universais, devido à baixa qualidade dessa vigilância e da resposta da saúde pública, por exemplo, em decorrência da descentralização de ações para distritos ou províncias, o que pode gerar a perda dos dados coletados localmente ${ }^{53}$.

Os países que adotaram sistemas sentinelas, como a França, o Reino Unido e os EUA (com assistência privada na APS), apesar de possuírem sistemas considerados eficazes para detectar grandes problemas de saúde pública, demonstraram pouca sensibilidade a eventos raros, como o surgimento precoce de uma nova doença, porque essas infecções podem surgir em qualquer parcela da população e requerem um monitoramento abrangente e integrado com todos os níveis de atenção à saúde.

Outro desafio interpretativo observado neste artigo reside em relacionar o modo de vida e a ocupação dos territórios em distintos países e seus respectivos modelos de atenção. As modelagens assistenciais hospitalocêntricas em países de alta renda, mesmo com sistemas de vigilância robustos, poderão impacto menos resolutivo em populações vulneráveis e que convivem em espaços densamente povoados, sobretudo, sem condições de manutenção do distanciamento social. Sistemas centrados no hospital tendem a ser menos vantajosos em populações mais vulneráveis e em países de renda baixa ou média, independentemente do tipo de vigilância e/ou do seu grau de integração à APS.

$\mathrm{Na}$ contramão, modelos centrados em APS abrangente e fortemente orientada à comunidade terão desfechos de vigilância mais eficientes na medida em que realizarem suas ações valendo-se da competência cultural acumulada e atuarem no espaço social com equipes multiprofissionais e, muitas vezes, trabalhadores comunitários. Neste caso, a integração da vigilância à APS catalisa o desempenho de todo o sistema de saúde e minimiza os efeitos adversos da pandemia, mesmo e, principalmente, em territórios cuja proteção social ainda é residual.

Nas últimas duas décadas, outras abordagens visaram preencher essa lacuna, a exemplo da utilização dos dados de sites de notícias online, 
serviços de agregação de notícias, redes sociais e pesquisas na web, adotados por países como Austrália, Coreia do Sul, Singapura e coortes de comunidades longitudinais participativas presentes nas experiências da Índia e do Reino Unido ${ }^{54}$. A vigilância digital utiliza tecnologias para apoiar a inteligência epidemiológica, ativa ou passiva, por meio de plataformas digitais que reúnem, online, conjuntos de dados que permitem identificar casos e grupos de infecções, rastrear rapidamente contatos, monitorar padrões de viagem durante os bloqueios e habilitar mensagens de saúde pública em escala ${ }^{55,56}$.

Cabe destacar que o uso mais abrangente de vigilância digital expõe outras preocupações, inclusive no que concerne à violação da privacidade, tanto durante quanto após o surto, pois nem todas as intervenções digitais incluíram a adoção consensual ou tornaram explícita a opção de consentimento do uso dos dados para fins específicos e por tempo determinado ${ }^{57}$.

Um exemplo sobre os aspectos supracitados é o uso de aplicativos em smartphones, pela Coreia do Sul, para divulgar os movimentos de pessoas com COVID-19, o que gerou temores de que tal ação pudesse levar as pessoas a evitarem a testagem (ou outras medidas impostas pelo governo), a fim de não sofrerem violação de sua privacida$\mathrm{de}^{25}$. Já em relação aos aplicativos de rastreamento de contato, uma fragilidade seria a necessidade de seu uso e obediência às orientações, por uma grande parcela da população, para que fosse eficaz na interrupção da transmissão comunitária ${ }^{58}$. Ademais, as principais questões práticas permanecem, como a compreensão de quais contatos são considerados próximos o suficiente para a transmissão e quando o tempo de exposição é considerado longo o suficiente para acionar um alerta.

Sendo assim, a vigilância digital pode gerar uma sensação de controle e ser vivenciada como um obstáculo à autonomia, com efeitos negativos sobre a motivação e o bem-estar ${ }^{59}$. Já o uso de sensores infravermelhos, incluindo câmeras de imagem térmica implantadas para identificar casos potenciais com base em sintomas febris (por exemplo, em aeroportos), pode gerar a falsa sensação de controle da situação, ante a ausência de sintomas. Outra preocupação óbvia é o grande número de resultados falso-positivos e falso-negativos, o que significa que é improvável que isso tenha um efeito substancial além de aumentar a conscientização $0^{60}$.

Painéis de dados estão sendo usados extensivamente na pandemia, coletando dados de saúde pública em tempo real, incluindo casos confirmados, mortes e números de testes, para manter o público informado e apoiar os formuladores de políticas no refinamento das intervenções. Os painéis ${ }^{61}$ COVID-19 normalmente se concentram em gráficos de série temporal e mapas geográficos, variando de estatísticas em nível de região a dados de coordenadas em nível de caso. Poucos painéis incluem uma análise dos dados sobre rastreamento de contatos ou vigilância da comunidade ${ }^{62}$.

Modelos matemáticos de epidemia têm sido amplamente usados para estimar a propagação e outros resultados de saúde da COVID-19, bem como a carga de doenças. Tais modelos dependem do conhecimento dos principais parâmetros de transmissão, como o intervalo serial (SI), o tempo entre o início dos sintomas do infectante e do infectado em uma cadeia de transmissão ${ }^{63-65}$ - que permite aproximar o intervalo de geração do tempo entre o momento da infecção de casos primários e casos secundários - e o número de reprodução variável no tempo (quantos casos secundários causados por um caso primário típico durante seu período de infecciosidade), durante o curso da pandemia ${ }^{59,66,67}$.

A qualidade e a consistência da coleta de dados permanecem uma preocupação. A falta de padrões oficiais e inconsistências nos relatórios governamentais de estatísticas entre os países tornam as comparações globais difíceis ${ }^{12}$ também em países com diferenças regionais marcantes, como Brasil, França e Canadá. Ademais, as estatísticas offline atualizadas e precisas dos governos nem sempre estão acessíveis de forma equitativa.

$\mathrm{Na}$ China, as políticas atuais e os sistemas de tecnologia têm limitações marcantes, que impedem a integração da atenção clínica e a APS, assim como a colaboração entre a APS e outros setores de atenção à saúde (por exemplo, hospitais), e tornam difícil garantir um número suficiente de profissionais com treinamento, remuneração e desempenho adequados na APS ${ }^{68}$.

Além disso, percebeu-se que os serviços de vigilância não podem operar isoladamente e precisam ser integradas aos sistemas públicos de saúde existentes. O uso de tecnologias digitais inovadoras e o compartilhamento de dados, apesar de potencializarem um efetivo controle, apresentaram limitações para o enfrentamento da pandemia de COVID-19, por constituírem intervenções verticais baseadas nas práticas tradicionais de notificação, registro e investigação de casos de doenças infecciosas e vigilância sindrômica, persistindo na consequente desarticulação em relação às 
ações desenvolvidas na atenção primária, que abarcam $80 \%$ dos casos mais leves da doença. Ao passo que as ações de vigilância à saúde abrangentes e multissetoriais, integradas com a APS, mostraram-se resolutivas e capazes de ofertar um monitoramento contínuo de todos os problemas de saúde que afetam a população local, inclusive, pós-pandemia. O sistema de vigilância integrado à APS contribuiu para o controle da transmissão na comunidade, a partir de intervenções direcionadas a orientar a implementação de medidas de mitigação na comunidade, incluindo escala, tempo, duração, estratégias específicas e relacionadas a demandas locais.

Nessa perspectiva, a vigilância participativa de doenças (PDS) também se mostrou uma estratégia efetiva para o monitoramento de doenças transmissíveis, na qual os cidadãos estão ativamente envolvidos no autorrelato de sintomas ou eventos, a fim de ajudar os especialistas em saúde pública a agregarem e analisarem dados para uma intervenção de saúde pública apropriada ${ }^{69}$. Ainda que, em sistemas de vigilância da atenção primária com base na participação voluntária, os resultados enviesados possam surgir pela falta de representatividade da população monitorada e da incerteza quanto ao denominador populacional.

A experiência cubana explicita o eixo comum para a integração organizacional e integração profissional da APS ao sistema de vigilância, para a análise dos problemas de saúde de forma ampliada, não apenas com foco na sistematização de in- dicadores gerais, mas com o propósito de subsidiar o planejamento e a organização de sistemas e serviços, o que exige um modelo de APS que contemple o conhecimento das condições de vida e trabalho das pessoas e das formas de organização e de atuação dos diversos órgãos governamentais e não governamentais, em cada território. Ou seja, um modelo de atenção articulado com os espaços de deliberação coletivos e representativos dentro da sociedade, e que se adapte a diferentes realidades, para fornecer ações integrais e equânimes $^{51,70,71}$.

O modelo de vigilância integrada à APS envolve a articulação de práticas de equipe multidisciplinar ou interprofissional, incluindo os prestadores de cuidados primários; um sistema de financiamento ou programa de incentivos para vigilância em saúde, promoção da saúde e prevenção de doenças; um sistema de tecnologia de informação para coleta de dados e instituição de planos e protocolos comuns, de forma contínua e sistemática; e a capacidade de detectar e relatar doenças novas e emergentes, por meio de interfaces articuladas entre os sistemas de saúde locais, provinciais, nacionais e internacionais.

Por fim, as experiências analisadas demonstraram que, embora fortes sistemas de vigilância sejam ferramentas indispensáveis para detecção e monitoramento de surtos e emergências de saúde pública, fortes sistemas de atenção primária formam a base de qualquer resposta a emergências sanitárias.

\section{Colaboradores}

Todos os autores contribuíram substancialmente na concepção e no planejamento do estudo; na obtenção, na análise e interpretação dos dados e na redação e revisão crítica e aprovaram a versão final a ser publicada. 


\section{Referências}

1. Hopman J, Allegranzi B, Mehtar S. Managing COVID-19 in Low- and Middle-IncomeCountries. JAMA 2020; 323(16):1549-1550.

2. Lloyd-Sherlock P, Ebrahim S, Geffen L, McKee M. Bearing the brunt of covid-19: older people in low and middle income countries. BMJ 2020 Mar 13; 368:m1052.

3. Tognotti E. Lessons from the History of Quarantine, from Plague to Influenza A. Emerg Infect Dis February 2013; 19 (2): 254-259.

4. Nussbaumer-Streit B, Mayr V, Dobrescu AI, Chapman A, Persad E, Klerings I, Wagner G, Siebert U, Christof C, Zachariah C, Gartlehner G. Quarantine alone or in combination with other public health measures to control COVID-19: a rapid review. Cochrane Database Syst Rev 2020; 4(4):CD013574.

5. Thacker SB, Berkelman RL. Public Health Surveillance in the United States. Epidemiologic Reviews 1988; 10:164-190.

6. Paim JS. Vigilância da saúde: tendências de reorientação de modelos assistenciais para a promoção da saúde. In: Czeresnia D, Freitas CM, organizadores. Promoção da saúde: conceitos, reflexões e tendências. Rio de Janeiro: Fiocruz; 2003.

7. Teixeira CF, Paim JS, Vilasboas AL. SUS, modelos assistenciais e vigilância da saúde. Inf Epidemiol SUS 1998; 7(2):7-28.

8. Rawaf S, Allen LN, Stigler FL, Kringos D, Quezada Yamamoto $\mathrm{H}$, van Weel C. Lessons on the COVID-19 pandemic, for and by primary care professionals worldwide. Eur J Gen Pract 2020; 26(1): 129-133.

9. Sarti TD, Lazarini WS, Fontenelle LF, Almeida APSC. Organization of Primary Health Care in pandemics: a rapid systematic review of the literature in times of COVID-19. Med Rxiv [Preprint] 2020.

10. Budd J, Miller BS, Manning EM, Lampos V, Zhuang M, Edelstein M, Rees G, Emery VC, Stevens MM, Keegan N, Short MJ, Pillay D, Manley E, Cox IJ, Heymann D, Johnson AM, McKendry RA. Digital technologies in the public health response to COVID-19. Nat Med 2020; 26:1183-1192.

11. Mei X, Lee HC, Diao K, et al. Artificial intelligence-enabled rapid diagnosis of COVID-19 patients. Med Rxiv [Preprint] 2020.

12. Thorlund K, Dron L, Park J, Hsu G, Forrest JI, Mills EJ. A real-time dashboard of clinical trials for COVID-19. Lancet Digit Health 2020 Jun; 2(6):e286-e287.

13. Menni C, Valdes AM, Freidin MB, Sudre CH, Nguyen LH, Drew DA, Ganesh S, Varsavsky T, Cardoso MJ, ElSayed Moustafa JS, Visconti A, Hysi P, Bowyer RCE, Mangino M, Falchi M, Wolf J, Ourselin S, Chan AT, Steves CJ, Spector TD. Real-time tracking of self-reported symptoms to predict potential COVID-19. Nat Med 2020; 26(7):1037-1040

14. Armitage H. Stanford Medicine scientists hope to use data from wearable devices to predict illness, including COVID-19. [cited 2020 Apr 14]. Stanford Medicine News Center [Internet]. Available from: http://med. stanford.edu/news/all-news/2020/04/wearable-devices-for-predicting-illness-.html
15. Okma KG, Marmor TR. Comparative studies and healthcare policy: learning and mislearning across borders. Clin Med (Lond) 2013; 13(5):487-491.

16. Calvo RA, Deterding S, Ryan RM. Health surveillance during covid-19 pandemic. BMJ 2020; 369:m1373.

17. French M, Monahan T. Dis-ease Surveillance: How Might Surveillance Studies Address COVID-19? Surveill Soc 2020; 18:1-11.

18. Nsubuga P, White ME., Thacker SB, Anderson MA, Blount SB, Broome CV, Chiller TB, Espitia V, Imtiaz R, Sosin D, Stroup DF, Tauxe V, Vijayaraghavan M, Trostle M. Vigilância em saúde pública: uma ferramenta para direcionar e monitorar atividades. In: Jamison DT, Breman J, Measham AR, Alleyne G, Claeson M, Evans D, Jha P, Mills A, Musgrove P. Priorities for disease control in developing countries. $2^{\mathrm{a}}$ ed. Washington, DC: Banco Mundial e Oxford University Press; 2006.

19. Qin L, Sun Q, Wang Y, Wu KF, Chen M, Shia BC, Wu SY. Prediction of number of cases of 2019 novel coronavirus (COVID-19) Using social media search index. Int J Environ Res Public Health 2020; 17(7):2365.

20. Sun K, Chen J, Viboud C. Early epidemiological analysis of the coronavirus disease 2019 outbreak based on crowdsourced data: a population-level observational study. Lancet Digit Health 2020; 2(4):e201-e208.

21. Gong M, Liu L, Sun X, Yang Y, Wang S, Zhu H. CloudBased System for Effective Surveillance and Control of COVID-19: Useful experiences from Hubei, China. J Med Internet Res 2020; 22(4):e18948.

22. Jao N, Cohen D, Udemans C. Technode. 2020. How China is using $Q R$ code apps to contain Covid-19. [Internet]. 25 de fevereiro. [cited 2020 Apr 8] Available from: https://technode.com/2020/02/25/how-chinais-using-qr-code-apps-to-contain-covid-19/

23. Kraemer MUG, Yang CH, Gutierrez B, Wu CH, Klein B, Pigott DM; Open COVID-19 Data Working Group, du Plessis L, Faria NR, Li R, Hanage WP, Brownstein JS, Layan M, Vespignani A, Tian H, Dye C, Pybus OG, Scarpino SV. The effect of human mobility and control measures on the COVID-19 epidemic in China. Science 2020; 368(6490):493-497.

24. Jia JS, Lu X, Yuan Y, Xu G, Jia J, Christakis NA. Population flow drives spatio-temporal distribution of COVID-19 in China. Nature 2020; 582(7812):389394.

25. Grind K, McMillan R, Wilde Mathews A. To Track Virus, Governments Weigh Surveillance Tools That Push Privacy Limits. The Wall Street Journal 2020 março 23. [cited 2020 Apr 8]. Available from: https:// www.wsj.com/\%0Darticles/to-track-virus-governments-weigh-surveillance-tools-that-push-privacylimits-11584479841

26. Oliver N, Lepri B, Sterly H, et al. Mobile phone data for informing public health actions across the COVID-19 pandemic life cycle. Sci Adv 2020; 6(23):eabc0764.

27. Lin RJ, Lee TH, Lye DCB. From SARS to COVID-19: the Singapore journey. Med J Aust 2020; 212(11): 497502. 
28. Park SY, Kim YM, Yi S, Lee S, Na BJ, Kim CB, Kim JI, Kim HS, Kim YB, Park Y, Huh IS, Kim HK, Yoon HJ, Jang H, Kim K, Chang Y, Kim I, Lee H, Gwack J, Kim SS, Kim M, Kweon S, Choe YJ, Park O, Park YJ, Jeong EK. Outbreak of coronavirus disease in Call Center, South Korea. Emerg Infect Diseases 2020; 26(8):16661670.

29. COVID-19 National Emergency Response Center, Epidemiology and Case Management Team, Korea Centers for Disease Control and Prevention. Early Epidemiological and Clinical Characteristics of 28 Cases of Coronavirus Disease in South Korea. Osong Public Health Res Perspect 2020; 11(1):8-14.

30. Outbreaks near me. [cited 2020 dec 28]. Available from: https://outbreaksnearme.org/us/en-US/

31. L'istituto Superiore Di Sanità. Informazioni Privacy Per La Sorveglianza Sanitaria Integrata Covid-19. [cited 2020 Mai 12]. Available from: https://www. epicentro.iss.it/coronavirus/pdf/informazioni-privacy-iss-sorveglianza-integrata-covid-19.pdf.

32. Pezzotti P, Punzo O, Bella A, Del Manso M, Urdiales AM, Fabiani M, Ciervo A, Andrianou X, Riccardo F, Stefanelli $P$. The challenges of the outbreak: the Italian COVID-19 integrated surveillance system. Eur J Public Health 2020; 30(Supl. 5):ckaa165.356.

33. Prodotto dall'Istituto Superiore di Sanità (ISS). Epidemia COVID-19 Aggiornamento nazionale 13 ottobre 2020. Roma, 13 ottobre 2020. [cited 2020 Oct 20]. Available from: https://www.epicentro.iss.it/ coronavirus/bollettino/Bollettino-sorveglianza-integrata-COVID-19_13-ottobre-2020.pdf.

34. COVID-19 National Incident Room Surveillance Team. COVID-19, Australia: Epidemiology Report 23 (Fortnightly reporting period ending 16 August 2020). Commun Dis Intell (2018) 2020; 44.

35. Australian Health Protection Principal Committee (AHPPC). Coronavirus (COVID-19) in Australia, Pandemic Health Intelligence Plan. [cited 2020 Maio 22]. Available from: https://www.health.gov. $\mathrm{au} /$ sites/default/files/documents/2020/05/coronaviruscovid-19-in-australia-pandemic-health-intelligence-plan_1.pdf

36. Ihekweazu C, Agogo E. Africa's response to COVID-19. BMC Med 2020; 18(1):51.

37. Kapata N, Ihekweazu C, Ntoumi F, Raji T, Chanda-Kapata P, Mwaba P, Mukonka V, Bates M, Tembo J, Corman V, Mfinanga S, Asogun D, Elton L, Arruda LB, Thomason MJ, Mboera L, Yavlinsky A, Haider N, Simons D, Hollmann L, Lule SA, Veas F, Abdel Hamid MM, Dar O, Edwards S, Vairo F, McHugh TD, Drosten C, Kock R, Ippolito G, Zumla A. Is Africa prepared for tackling the COVID-19 (SARS-CoV-2) epidemic. Lessons from past outbreaks, ongoing pan-African public health efforts, and implications for the future. Int I Infect Dis 2020; 93:233-236.

38. Siedner MJ, Harling G, Derache A, Smit T, Khoza T, Gunda R, Mngomezulu T, Gareta D, Majozi N, Ehlers E, Dreyer J, Nxumalo S, Dayi N, Ording-Jesperson G, Ngwenya N, Wong E, Iwuji C, Shahmanesh M, Seeley J. Protocol: Leveraging a demographic and health surveillance system for Covid-19 Surveillance in rural KwaZulu-Natal. Wellcome Open Res 2020; 5:109.
39. Lallukka T, Pietiläinen $\mathrm{O}$, Jäppinen $\mathrm{S}$, Laaksonen $\mathrm{M}$, Lahti J, Rahkonen O. Factors associated with health survey response among young employees: a register-based study using online, mailed and telephone interview data collection methods. BMC Public Health 2020; 20(1):184.

40. Senegal. A Promising practice on multisectoral communication efforts in Senegal Phcpi. CoviD-19 Promisi N G Practices. [cited 2020 Apr 18]. Available from: https://improvingphc.org/sites/default/files/Senegal_ Risk\%20Communication.pdf

41. Garg S, Bhatnagar N, Gangadharan N. A Case for Participatory Disease Surveillance of the COVID-19 Pandemic in India. JMIR Public Health Surveill 2020; 6(2):e18795.

42. Singh S. Financial Express. 2020. Aarogya Setu is Government of India's first 'comprehensive' COVID-19 tracking app, here are all the details. [cited 2020 Apr 18]. Available from: https://www.financialexpress.com/ industry/technology/aarogya-setu-is-government -of-indias-first-comprehensive-covid-19-trackingapp-here-are-all-the -details / 1916887 /.

43. de Lusignan S, Jones N, Dorward J, Byford R, Liyanage H, Briggs J, Ferreira F, Akinyemi O, Amirthalingam G, Bates C, Lopez Bernal J, Dabrera G, Eavis A, Elliot AJ, Feher M, Krajenbrink E, Hoang U, Howsam G, Leach J, Okusi C, Nicholson B, Nieri P, Sherlock J, Smith G, Thomas M, Thomas N, Tripathy M, Victor W, Williams J, Wood I, Zambon M, Parry J, O’Hanlon S, Joy M, Butler C, Marshall M, Hobbs FDR. The Oxford Royal College of General Practitioners Clinical Informatics Digital Hub: Protocol to Develop Extended COVID-19 Surveillance and Trial Platforms. JMIR Public Health Surveill 2020; 6(3):e19773.

44. Turbelin C, Boëlle PY. Improving general practice based epidemiologic surveillance using desktop clients: the French Sentinel Network experience. Stud Health Technol Inform 2010; 160(Pt 1):442-446.

45. Public Health England. Weekly Coronavirus Disease 2019 (COVID-19) Surveillance Report Summary of COVID-19 surveillance systems. [cited 2020 Ago 8]. Available from: https://assets.publishing.service.gov. uk/government/uploads/system/uploads/attachment_data/file/888254/COVID19_Epidemiological_ Summary_w22_Final.pdf.

46. Arenas A, Cota W, Gómez-Gardeñes J, Gómez S, Granell C, Matamalas JT, Soriano D, Steinegger B. A mathematical model for the spatiotemporal epidemic spreading of COVID19. [preprint] Med Rxiv 2020. [cited 2020 Ago 8]. Available from: https://doi. org/10.1101/2020.03.21.20040022

47. Czubaj F. Coronavirus en la Argentina: las clínicas privadas podrán hasta 7500 tests diarios for descomprimir el sistema de salud público. La Nación; 2020 Abr 28.

48. Vales L. Coronavirus: el operativo detectar estrena protocolo para todo el país. 25 de mayo de 2020. [cited 2020 Jul 12]. Available from: https://www.pagina12.com.ar/268006-coronavirus-el-operativo-detectar-estrena-protocolo-para-tod.

49. Gemelli, N. Management of the COVID-19 outbreak in Argentina: the beginning. Disaster Medicine and Public Health Preparation 2020; 1-3. 
50. Gorry C. COVID-19 case detection: Cuba's active screening approach. Medical Review 2020; 22(2).

51. Aguilar-Guerra, Tania L Reed, Gail. Mobilization of primary health care: Cuba's powerful weapon against COVID-19. Medical Review 2020; 22(2):58-63.

52. Pérez Riverol A. The Cuban Strategy for Combatting the COVID-19 Pandemic. MEDICC Review 2020; 22(3):64-68. [cited 2020 Out 19]. Available from: http://search.ebscohost.com/login.aspx?direct $=$ true $\& \mathrm{db}=\mathrm{c} 8 \mathrm{~h} \& \mathrm{AN}=144979497 \&$ lang $=\mathrm{pt}-\mathrm{br} \&$ site $=$ eds-live.

53. Randazzo W, Cuevas-Ferrando E, Sanjuán R, Domingo-Calap P, Sánchez G. Metropolitan wastewater analysis for COVID-19 epidemiological surveillance. Int J Hyg Environ Health 2020; 230:113621.

54. Ricoca PV, Nunes C, Abrantes A. Vigilância epidêmica de Covid-19: considerando a incerteza e subavaliação. Karger AG, Basel (2020), pp. 1-7.

55. Lodder W, de Roda Husman AM. SARS-CoV-2 in wastewater: potential health risk, but also data source. Lancet Gastroenterol Hepatol 2020; S24681253(20)30087-X.

56. Nsubuga P, Nwanyanwu O, Nkengasong JN, Mukanga D, Trostle M. Strengthening public health surveillance and response using the health systems strengthening agenda in developing countries. BMC Public Health 2010; 10 (Supl. 1):S5-S6

57. Ienca M, Vayena E. On the responsible use of digital data to tackle the COVID-19 pandemic. Nat Med 2020; 26:463-464.

58. Ferretti L, Wymant C, Kendall M, Zhao L, Nurtay A, Abeler-Dörner L, Parker M, Bonsall D, Fraser C. Quantifying SARS-CoV-2 transmission suggests epidemic control with digital contact tracing. Med Rxiv [Preprint] 2020.

59. Jensen JM, Raver JL. When Self-Management and Surveillance Collide: Consequences for Employees' Organizational Citizenship and Counterproductive Work Behaviors. Group \& Organization Management 2012:37(3).

60. Gostic K, Gomez AC, Mummah RO, Kucharski AJ, Lloyd-Smith JO. Estimated effectiveness of symptom and risk screening to prevent the spread of COVID-19. Elife 2020; 9:e55570.

61. Lampos V, Majumder MS, Yom-Tov E, Edelstein M Moura S, Hamada Y, Rangaka MX, McKendry RA, Cox IJ. Tracking COVID-19 using online search. npj Digit. Med. 4, 17 (2021). https://doi.org/10.1038/ s41746-021-00384-w

62. McKendry RA, Rees G, Cox IJ, Johnson A, Edelstein M, Eland A, Stevens MM, Heymann D. Share mobile and social-media data to curb COVID-19. Nature 2020 Apr; 580(7801):29.

63. Buckee CO, Balsari S, Chan J, Crosas M, Dominici F Gasser U, Grad YH, Grenfell B, Halloran ME, Kraemer MUG, Lipsitch M, Metcalf CJE, Meyers LA, Perkins TA, Santillana M, Scarpino SV, Viboud C, Wesolowski A, Schroeder A. Aggregated mobility data could help fght COVID-19. Science 2020: 368(6487):145-146.

64. Black AJ, Ross JV. Estimating a markovian epidemic model using household serial interval data from the early phase of an epidemic. PLoS One 2003; 8(8): e73420.
65. Yom-Tov E, Johansson-Cox I, Lampos V, Hayward AC. Estimating the secondary attack rate and serial interval of influenza like illnesses using social media. Influenza and Other Respiratory Viruses 2015; 9(4):191-199.

66. Zhou L, Li Q, Uyeki TM. Estimated incubation period and serial interval for human-to-human influenza A(H7N9) virus transmission. Emerging Infectious Diseases 2019; 25(10):1982-1983.

67. Smolinski MS, Crawley AW, Olsen JM, Jayaraman T, Libel M. Participatory disease surveillance: engaging communities directly in the notification, monitoring and response to health threats. JMIR Public Health Surveill 2017 October 11; 3(4):e62.

68. Xi Li, Jiapeng Lu, Shuang Hu, KK Cheng, Jan De Maeseneer, Qingyue Meng et al.. The primary health-care system in China. Lancet 2017; 390 (10112):2584-2594.

69. Souty C, Turbelin C, Blanchon T, Hanslik T, Le Strat $\mathrm{Y}$, Boëlle PY. Improving disease incidence estimates in primary care surveillance systems. Popul Health Metr 2014 Jul 26; 12:19.

70. Greenhalgh T, Koh GCH, Car J. Covid-19: a remote assessment in primary care. BMJ 2020 Mar 25; 368:m1182.

71. Stevenson Rowan, M., Hogg, W., \& Huston, P. (2007). Integrating public health and primary care. Politiques de Sante 2007; 3(1):e160-e181.

Artigo apresentado em 22/10/2020

Aprovado em 27/03/2021

Versão final apresentada em 29/03/2021

Editores-chefes: Romeu Gomes, Antônio Augusto Moura da Silva 
